\title{
Estrategias para proteger al recién nacido y lactante menor de 6 meses de la coqueluche. Posición del Comité Consultivo de Inmunizaciones de la Sociedad Chilena de Infectología (CCI)
}

\author{
Marcela Potin, Alberto Fica, Liliana Véliz, Gabriela Moreno, Jan Wilhelm y Jaime Cerda, en representación del CCI
}

Strategies to protect the newborn and infants under 6 months of age against pertussis. Statement of the Advisory Committee for Immunizations of the Chilean Infectious Diseases Society

In recent years there have been Pertussis outbreaks not seen in the last 50 years affecting adults, adolescents and children and causing deaths in young unvaccinated infants. In Chile an outbreak of Pertussis started in year 2011, leaving 16 infants less than 3 months dead during this year, twice the number seen in a non epidemic year. These children were infected before receiving the programmatic vaccines indicated at 2, 4 and 6 months of age, usually from close contacts, especially their mothers. Pertussis control has not been possible for several reasons, such as limited immunity duration of available vaccines and their poor impact on nasopharyngeal carriage, situation that keeps the agent's circulation and transmission, condition often asymptomatic or unrecognized. Additionally, the use of acellular vaccines appears to be a determining factor because they induce an immune response with poor immune memory and consequently a short time duration. The acellular vaccines with reduced antigen content, available for adolescents and adults, has allowed the evaluation of various strategies but none has succeeded in reducing infant mortality. Recently a new strategy of vaccinating pregnant women against Pertussis in the second or third semester has shown remarkable results reducing up to $90 \%$ infant deaths due to Pertussis infection. This strategy prevents mother's infection avoiding child infection through respiratory droplets and also provides the child with antibodies from placental transmission. Improved pertussis vaccines are required, in the meantime the Committee considers that the pregnant immunization strategy, between the 27 and 36 weeks, with acellular pertussis vaccine should be included in our national vaccine program.

Key words: Pertussis infection, whooping cough, maternal immunization.

Palabras clave: Coqueluche, tos ferina, vacunación materna.

\section{Situación epidemiológica}

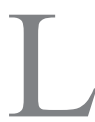

a coqueluche o tos convulsiva, constituye junto al sarampión, una de las dos enfermedades inmu-

noprevenibles de mayor transmisibilidad, siendo cada una de ellas capaz de enfermar cerca de 12 a 17 personas susceptibles. A pesar del uso de vacunas eficaces por más de 60 años, la coqueluche continúa siendo un problema de salud pública provocando anualmente unas 200.000 muertes de lactantes en el mundo ${ }^{1}$. En forma cíclica, cada dos a cinco años ocurren brotes epidémicos, incluso en países con altas coberturas de vacunación y entre los años 2010 y 2012 se alcanzaron las incidencias más altas de los últimos 20 a 50 años en países desarrollados como E.U.A. ${ }^{2}$, Australia ${ }^{3}$, y Reino Unido ${ }^{1}$. Este problema también se observó en países en vías de desarrollo como Argentina, Brasil, Colombia y Chile ${ }^{4}$. En nuestro país, el brote se inició el año 2011 y se observó más intensamente en regiones densamente pobladas como la Región del Bío-Bío, Valparaíso y en la Región Metropolitana ${ }^{5}$. Estos aumentos de la incidencia afectan a lactantes bajo 6 meses de edad, que por su edad no han iniciado la vacunación o no la han completado o tampoco poseen ya inmunidad pasiva transferida desde la madre. Los brotes también afectan a escolares o adolescentes y adultos, en los que la inmunidad, ya sea natural o por vacunas se ha desvanecido con el paso del tiempo ${ }^{6}$, constituyéndose en el reservorio infectante para el lactante menor. La relevancia de estos brotes queda subrayada por el hecho de que en Chile el año del inicio del brote el 2011 se registraron 16 muertes por coqueluche, todas ellas en lactantes bajo 6 meses de edad $^{5}$. Sin embargo, en períodos no epidémicos como los años 2014 y 2015 igualmente fallecieron 7 y 8 lactantes cada año, respectivamente ${ }^{7}$.

\section{Causas de los brotes}

Las razones para el insuficiente control de la enfermedad son varias y complejas e incluyen por un lado la duración limitada de la protección que otorgan las vacunas
Conflictos de interés: L. Véliz y M. Potin han recibido honorarios por charlas en reuniones científicas organizadas por Merck Sharp \& Dohme (MSD), J. Wilhelm ha recibido ayuda por parte de Pfizer para asistir a un Congreso y honorarios por parte de GSK.

Recibido: 5 de octubre de 2016

Correspondencia a: Marcela Potin Santander mpotin@med.puc.cl 
que va de 4 a 12 años según diferentes estudios ${ }^{3,8-10}$, así como su incapacidad para interrumpir la circulación de Bordetella pertussis ${ }^{11,12}$ dado su escaso efecto en portación nasofaríngea del agente. Así mismo, es probable que la presión selectiva que ejercen las vacunas, esté favoreciendo la emergencia de cepas mutantes del microorganismo, con mejores mecanismos patogénicos que logren infectar a individuos vacunados ${ }^{13-16}$. Otros factores que también pueden contribuir son el aumento de la sospecha clínica y disponibilidad de nuevas técnicas diagnósticas como la reacción de polimerasa en cadena (RPC) en tiempo real, de alta sensibilidad y especificidad. El deterioro en las coberturas de vacunas en algunas regiones puede estar también influyendo. La importancia de cada uno de estos factores en los brotes, probablemente varía dentro de una región a otra e incluso en un mismo país, pero existe consenso de que el tipo vacuna anti-coqueluche aplicada es un fuerte determinante en la resurgencia de la enfermedad.

\section{Vacunas disponibles: celulares y acelulares}

Existen dos tipos de vacunas anti-pertussis, las llamadas celulares y las acelulares. Las primeras son fabricadas en base a bacterias (células) completas inactivadas y fueron introducidas en la década de 1950. Sólo se indican en niños bajo 7 años de edad ya que se asocian a una mayor cantidad de reacciones adversas en grupos de más de 6 años. En contraste, las vacunas llamadas acelulares se desarrollan e introducen a partir de 1970, por temores a dichas reacciones adversas. Estas últimas se componen de dos a cinco antígenos altamente purificados y producen menos reacciones locales y sistémicas, pero su desventaja es que despiertan escasa memoria inmunológica por lo que la duración de su protección es aún más breve (2-5 años) y requieren de refuerzos frecuentes ${ }^{17}$. Existen vacunas acelulares pediátricas y otras acelulares para uso en niños sobre los 6 años o adultos, de contenido antigénico reducido. Todas las vacunas disponibles no reducen la portación nasofaríngea de $B$. pertussis por lo que la circulación del microorganismo se puede mantener en algún grado a pesar de alcanzarse buenas coberturas.

\section{Estrategias propuestas}

Se reconoce la necesidad urgente de disponer de mejores vacunas, pero mientras ello no ocurra, la morbimortalidad en lactantes bajo 3 meses de edad continuará, por lo que se han diseñado diferentes alternativas de solución, con un impacto variable. Se sabe que la fuente de infección de estos niños son predominantemente sus padres $y / o$ hermanos ${ }^{18,19}$ y la enfermedad los afecta en un período en el que es imposible que aún estén protegidos por vacunas. Así, para controlar el problema se ha usado la vacunación del entorno del niño o "estrategia capullo", con vacunas acelulares de contenido antigénico reducido (dTpa). En esta estrategia se inmuniza a la madre durante el puerperio así como al padre y los contactos cercanos mayores de 12 años. Esta estrategia fue utilizada en Chile en el brote de coqueluche de los años 2011 y 2012 en algunas regiones como Valparaíso, Bío-Bío, Metropolitana y del Libertador B. O'Higgins. Las tasas de incidencia por $B$. pertussis bajo 6 meses de edad, aumentaron de $166 / 100.000$ en el año 2010 , a 503/100.000 en el 2011 y $910 / 100.000$ el año 2012. En conjunto con otras medidas eliminó las muertes de lactantes por coqueluche mientras se utilizó ${ }^{5}$, disminuyendo la tasa a 344/100.000 en los lactantes bajo 6 meses de edad. Su implementación, sin embargo, es reconocida como engorrosa y difícil de mantener en el tiempo. Si bien la estrategia capullo logró inmunizar a $91 \%$ de las madres en el puerperio en Chile, sólo pudo cubrir a $60 \%$ de los otros contactos del niño ${ }^{5}$. Adicionalmente, dado que la respuesta inmune a la vacuna demora al menos dos semanas, esta medida genera un período de ventana en que el recién nacido y lactante queda expuesto a la infección. Una segunda opción es administrar la vacuna dTpa a la mujer embarazada reduciendo por un lado el riesgo de infección en la madre y por otra parte protegiendo en forma pasiva al niño a través del traspaso de anticuerpos placentarios. Se ha demostrado que las concentraciones de anticuerpos que alcanzan los hijos de madres vacunadas, a los 2 meses, es significativamente superior a los de hijos de madres no vacunadas ${ }^{20,21}$. Por razones de seguridad, se ha analizado la posible interferencia de esta medida con la respuesta inmune de las vacunas pediátricas habituales del primer semestre de vida del niño y los datos señalan una reducción discreta a moderada de los niveles de anticuerpos para difteria y toxina pertussis en hijos de madres vacunadas ${ }^{21,22}$. Aun así se alcanzan concentraciones de anticuerpos protectoras para $H$. influenzae $b$, tétanos, difteria y hepatitis B y la interferencia desaparece después de las dosis de refuerzo en el segundo año de vida ${ }^{21,23}$. La seguridad de esta estrategia queda además respaldada por los datos epidemiológicos provenientes de aquellos países que ya han adoptado esta estrategia en los últimos tres a cuatro años, donde no se ha visto un resurgimiento de otras enfermedades infecciosas prevenibles por vacunas. La evidencia de la efectividad de vacunar a la mujer embarazada proviene de los estudios observacionales y de la vigilancia epidemiológica en el Reino Unido, región que incorporó la vacuna dTpa en el embarazo en el año 2012. Los datos señalan una efectividad cercana a $90 \%$ para prevenir muertes por tos ferina en lactantes bajo 2 meses de edad ${ }^{24,25}$ y un impacto positivo en la disminución del número de casos confirmados y hospitalizaciones, especialmente en el grupo de lactantes bajo dos meses. Estas cifras indican que el beneficio de evitar muertes 
por coqueluche bajo 3 meses de edad supera los riesgos potenciales de algún grado de interferencia con vacunas recibidas por el lactante luego de nacer. Esta intervención adicionalmente se acompaña de concentraciones significativamente más altas de anticuerpos anti-pertussis en la leche materna de mujeres vacunadas ${ }^{26}$.

La mejor edad de vacunación sería ente las 27 y 36 semanas de gestación, debido a que el traspaso de anticuerpos por la placenta es óptimo. En E.U.A. adicionalmente, se recomienda revacunar a la madre en cada nuevo embarazo $^{27,28}$ dado que la declinación de anticuerpos en la madre es muy rápida. El uso de esta estrategia ha debido sortear el hecho de que no existe registro sanitario para la vacuna dTpa en el embarazo debido a que la industria farmacéutica no realiza ensayos de vacunas durante la gestación. Ello no ha impedido su incorporación en numerosos países con excelentes resultados.

En relación a la seguridad de esta vacuna, los toxoides tetánicos (TT) y diftérico (TD) ya se han utilizado por años en mujeres embarazadas en todo el mundo para prevenir el tétanos neonatal demostrando su seguridad para la madre y el feto. La vacuna dTpa se ha utilizado en el embarazo y ha sido bien tolerada y no se ha asociado a complicaciones en la gestación, en el feto ${ }^{29}$, o el recién nacido ${ }^{30-33}$. Asimismo, en los seguimientos de lactantes hijos de madres vacunadas no se han observado trastornos del desarrollo psicomotor o del crecimiento de los niños ${ }^{30-34}$.

Actualmente, la vacunación de la mujer embarazada con dTpa es la estrategia en uso en E.U.A., Nueva Zelandia, Bélgica, Israel y el Reino Unido. También se aplica en algunos países de Latinoamérica como Argentina, Uruguay, Colombia, Costa Rica y México. Esta estrategia ha demostrado ser la más costo efectiva ${ }^{35}$ y es la recomendada actualmente por el Global Pertussis Initiative ${ }^{36}$ aunque ellos la sugieren sólo en situación de brotes. Los antecedentes presentados indican que puede ser una buena iniciativa para prevenir las muertes por coqueluche en lactantes bajo 3 meses de edad en nuestro país.

En resumen, el Comité Asesor de Vacunas de la Sociedad Chilena de Infectología recomienda:

- La vacunación de todas las mujeres embarazadas contra el coqueluche con una vacuna acelular aplicada entre las 27 y 36 semanas de gestación, sea en situación endémica o en caso de brote.

- Mantener la adherencia al esquema de vacunación del lactante en especial para la dosis de los 2 meses.

- Realizar los máximos esfuerzos para mantener altas coberturas de vacunación idealmente sobre $95 \%$ para la tercera dosis de vacuna pertussis.

- Aumentar la cobertura de técnicas diagnósticas de laboratorio para la confirmación de casos, con alta sensibilidad y especificidad como la RPC-en tiempo real en vez de la IFD actualmente en uso en Chile.

- No es posible por ahora resolver una recomendación sobre necesidad de nuevas dosis de vacuna acelular en cada gestación.

- El esquema de vacunación del lactante, deberá seguir según lo establecido en el Programa Nacional de Inmunizaciones.

\section{Resumen}

En los últimos años se han observado brotes de coqueluche no vistos en 50 años, afectando a adultos, adolescentes y escolares y provocando muertes en lactantes pequeños aún no vacunados. En Chile, en 2011 se inicia un brote con 16 lactantes bajo 3 meses de edad, fallecidos ese año el doble de muertes de un año no epidémico. Estos niños se infectan antes de recibir las vacunas programáticas de los 2, 4 y 6 meses de edad, a partir de contactos cercanos, en especial de su propia madre. El control de la coqueluche no ha sido posible por varias razones, como la duración acotada de la inmunidad de las vacunas disponibles y su escaso impacto en la portación nasofaríngea que mantiene la circulación y trasmisión del agente, con frecuencia en forma asintomática o no reconocida. Adicionalmente, el uso de las vacunas acelulares pareciera ser un factor determinante pues inducen una respuesta inmune con una pobre memoria inmunológica y por ello de duración más breve. La disponibilidad de vacunas acelulares de contenido antigénico reducido para adolescentes y adultos ha permitido ensayar diversas estrategias, pero ninguna ha logrado reducir la mortalidad de los lactantes. Más recientemente se ha ensayado la vacunación de la madre en el $2^{\circ}$ o $3^{\text {er }}$ trimestre del embarazo con resultados notables pues ha disminuido en más de $90 \%$ las muertes de lactantes por coqueluche. Esta medida evita la infección de la madre y con ello su transmisión por vía respiratoria al niño y permite transferir anticuerpos por vía placentaria. En espera de mejores vacunas, este comité considera que la inmunización de la mujer embarazada, idealmente entre las 27 y 36 semanas, con vacuna anti-coqueluche acelular debiera ser incluida en nuestro país en forma programática.

\section{Referencias bibliográficas}

1.- Tan T, Dalby T, Forsyth K, Halperin S A, Heininger U, Hozbor D, et al, Pertussis across the globe. Recent epidemiologic trends from 2000 to 2013. Pediatr Infect Dis J 2015; 34 :
222-32.

2.- Pertussis (whooping cough): pertussis outbreak trends. Centers for Disease Control and Prevention Web site. Available at: http:// www.cdc.gov/pertussis/outbreaks/trends.html. (Revisado el 20 de julio de 2016).
3.- Quinn H E, Snelling T L, Macartney K K, McIntyre P B. Duration of protection after first dose of acelullar pertussis vaccine in infants. Pediatrics 2014; 133 (3): e513-9 doi:10.1542/ peds.2013-3181.

4.- OPS Alerta epidemiológica, tos ferina del 16 
de noviembre de $2012 \mathrm{http} / / /$ www.paho.org/hq/ index.php?option $=$ com_docman\&task $=$ doc_vie w\&Itemid $=270 \&$ gid $=19326 \&$ lang=es $($ Revisado el 11 de julio de 2016).

5.- Tos ferina o coqueluche situación epidemiológica. Dpto. Epidemiologia, DEIS, MINSAL (Revisado agosto de 2013).

6.- Wendelboe A M, Van Rie A, Salmaso S, et al. Duration of immunity against pertussis after natural infection or vaccination. Pediat Infect Dis J 2005; 24: S58-S61.

7.- Tos ferina o coqueluche situación epidemiológica, enero-diciembre de $2015 \mathrm{http}: / /$ epi.minsal.cl/wp-content/uploads/2016/03/ Informe-Anual-Coqueluche-2015.pdf. (Revisado el 2 de julio de 2016).

8.- Misegades L K, Winter K, Harriman K, Talarico J, Messonnier N E, Clark T A, et al. Association of childhood pertussis with receipt of 5 doses of pertussis vaccine by time since last vaccine dose, California, 2010. JAMA. 2012; 308: 2126-32. doi: 10.1001/jama.2012.14939.

9.- Jenkinson D. Duration of effectiveness of pertussis vaccine: evidence from a 10 year community study. Br Med J 1988; 296: 612-4.

10.- Cattaneo L A, Reed G W, Haase D H, et al. The seroepidemiology of Bordetella pertussis infections: a study of persons ages 1-65 years. J Infect Dis 1996; 173: 1256-9.

11.- Mertsola J, Ruuskanen O, Eerola E, Viljanen M K. Intrafamilial spread of pertussis. J Pediatr 1983; 103: 359-63.

12.- Long S S, Welkon C J, Clark J L. Widespread silent transmission of pertussis in families: antibody correlates of infection and symptomatology. J Infect Dis 1990; 161: 480-6.

13.- Mooi F R, Van der Maas N A, De Melker H E. Pertussis resurgence: waning immunity and pathogen adaptation-two sides of the same coin. Epidemiol. Infect 2014; 142: 685-94. doi: 10.1017/S0950268813000071. Epub $2013 \mathrm{Feb}$ 13.

14.- Safarchi A, Octavia S, Wai Luu L D, Tai C Y, Sintchenko V, Wood N, et al. Better colonization of newly emerged Bordetella pertussis in the co-infection mouse model study. Vaccine 2016; 34: 3967-71dx.doi.org/10.1016/j. vaccine.2016.06.052.

15.- Hegerle N, Paris A S, Brun D, Dore G, Njamkepo E, Guillot S, et al. Evolution of French Bordetella pertussis and Bordetella parapertussis isolates: increase of Bordetellae not expressing pertactin. Clin Microbiol Infect 2012; 18: E40-E46. doi: 10.1111/j.14690691.2012.03925.x. Epub 2012 Jun 21.

16.- Barkoff A-M, Mertsola J, Guillot S, Guiso N, Berbers G, He Q. Appearance of Bordetella pertussis strains not expressing the vacccine antigen pertactin in Finland. Clin Vaccine Immunol 2012; 19: 1703-4. Epub 2012 Aug 22. 17.- Witt M A, Katz P H, Witt D J. Unexpectedly limited durability of immunity following acellular pertussis vaccination in preadolescents in a North American outbreak. Clin Infect Dis 2012: 54: 1730-5. doi: 10.1093/cid/cis287. Epub 2012 Mar 15.

18.- Schellekens J, Wirsing von Konig C-H, MD, Gardner P Pertussis sources of infection and routes of transmission in the vaccination era. Pediatr Infect Dis J 2005; 24: S19-S24.

19.- Perret C, Viviani T, Peña A M, Abarca K, Ferres M. Fuente de infección de Bordetella pertussis en lactantes hospitalizados por tos ferina. Rev Med Chile 2011; 139: 448-54

20.- Gall S. Prevention of pertussis, tetanus and diphtheria among pregnant, post partum women and infants. Clin Obstet Gynecol 2012; 55: 498509. doi: 10.1097/GRF.0b013e31824f3b38.

21.- Hardy-Fairbanks A J, Pan S J, Decker M D, Johnson D R, Greenberg D P, Kirkland KB, et al. Immune responses in infants whose mothers received Tdap vaccine during pregnancy. Pediatr Infect Dis J 2013; 32: 1257-60.

22.- Jones C, Pollock L, Barnett S M, Battersby A, Kampmann B. The relationship between concentration of specific antibody at birth and subsequent response to primary immunization. Vaccine 2014; 32: 996-1002. doi: 10.1016/j. vaccine.2013.11.104. Epub 2013 Dec 14.

23.- Maertens K, Caboré R N, Huygen K, Vermeiren S, Hens N, Van Damme P, et al. Pertussis vaccination during pregnancy in Belgium: Follow-up of infants until 1 month after the fourth infant pertussis vaccination at 15 months of age. Vaccine 2016; 34: 3613-9. doi: 10.1016/j.vaccine.2016.04.066. Epub 2016 Apr 30.

24.- Amirthalingam G, Andrews N, Campbell H, Ribeiro S, Kara E, Donegan K, et al. Effectiveness of maternal pertussis vaccination in England: an observational study. Lancet.2014; 384: 1521-8. doi: 10.1016/S01406736(14)60686-3. Epub 2014 Jul 15.

25.- Dabrera G, Amirthalingam G, Andrews N, Campbell H, Ribeiro S, Kara E, et al. A casecontrol study to estimate the effectiveness of maternal pertussis vaccination in protecting newborn infants in england and Wales, 20122013. Clin Infect Dis 2015; 60: 333-7. doi: 10.1093/cid/ciu821. Epub 2014 Oct 19.

26.- AbuRaya B, Srugo I, Kessel A, Peterman M, Bader D, Gonen R, et al. The effect of timing of maternalseptetanus, diphtheria, and acellular pertussis (Tdap) immunization during pregnancy on newborn pertussis antibody levels-a prospective study. Vaccine 2014; 32 : 5787-93. doi: 10.1016/j.vaccine.2014.08.038. Epub 2014 Aug 28.

27.- Advisory Committee on Immunization Practices. Updated recommendations for use of tetanus toxoid, reduced diphtheria toxoid and acellular pertussis vaccine (Tdap) in pregnant women and persons who have or anticipate having close contact with an infant aged $<12$ months. MMWR Morb Mortal Wkly Rep 2011; 60: 1424-6.

28.- Centers for Disease Control and Prevention. Advisory Committee on Immunization Practices (ACIP) updated recommendations for use of tetanus toxoid, reduced diphtheria toxoid, and acellular pertussis vaccine (Tdap) in pregnant women-ACIP, 2012. MMWR Morb Mortal Wkly Rep 2013; 62: 131-5.

29.- Donegan K, King B, Bryan P. Safety of pertussis vaccination in pregnant women in UK: observational study. Br Med J. 2014 Jul 11; 349: g4219. doi: 10.1136/bmj.g4219.

30.- Muñoz F M, Bond N H, Maccato M, Pinell P, Hammill H A, Swamy G K, et al. Safety and immunogenicity of tetanus diphtheria and acellular pertussis (Tdap) immunization during pregnancy in mothers and infants: a randomized clinical trial. JAMA 2014; 311: 1760-9. doi:10.1001/jama.2014.3633.

31.- Zheteyeva Y A, Moro P L, Tepper N K, Rasmussen S A, Barash F E, Revzina N $\mathrm{V}$, et al. Adverse event reports after tetanus toxoid, reduced diphtheria toxoid, and acellular pertussis vaccines in pregnant women. Am J Obstet Gynecol 2012; 207 (1): 59 e1-7. doi: 10.1016/j.ajog.2012.05.006. Epub 2012 May 14.

32.- Moro P L, Cragan J, Tepper N, Zheteyeva Y, Museru O, Lewis P, et al. Enhanced surveillance of tetanus toxoid, reduced diphtheria toxoid, and acellular pertussis (Tdap) vaccines in pregnancy in the Vaccine Adverse Event Reporting System (VAERS), 2011-2015. Vaccine 2016: 24: 2349-53. doi: 10.1016/j. vaccine.2016.03.049. Epub 2016 Mar 22.

33.- Kharbanda E O, Vázquez-Benítez G, Lipkind H S, Klein N P, Cheetham T C, Naleway A, et al. Evaluation of the association of maternal pertussis vaccination with obstetric events and birth outcomes. JAMA. 2014; 312: 1897-1904. doi: 10.1001/jama.2014.14825.

34.- Englund J A, Anderson E L, Reed G F, Decker M D, Edwards K M, Pichichero M E, et al. The effect of maternal antibody on the serologic response and the incidence of adverse reactions after primary immunization with acellular and whole-cell pertussis vaccines combined with diphtheria and tetanus toxoids. Pediatrics 1995; 96 (3 pt 2): 580-4.

35.- Terranella A, Asay G R, Messonnier M L, Clark T A, Liang J L. Pregnancy dose Tdap and postpartum cocooning to prevent infant pertussis: A decision analysis. Pediatrics. 2013; 131(6): e1748-56. Available at: www.pediatrics. org/cgi/content/full/131/6/e1748.

36.- Forsyth K, Plotkin S, Tan T, Wirsing von König CH. Strategies to decrease pertussis transmission to infants. Pediatrics 2015 Jun; 135 (6): e1475-82 DOI: 10.1542/peds.2014-3925. 